Butwal Campus Journal, Vol. 3, No. 1: 103 -112, July 2020

Research Management Cell, Butwal Multiple Campus, Tribhuvan University, Nepal

\title{
ON SOME NEW RESULTS IN M-FUZZY METRIC SPACE
}

\author{
Thaneshwor Bhandari* Narayan Pahari ${ }^{\#}$ \\ *Associate Professor, Butwal Multiple Campus, Butwal, TU \\ * Professor, Central Department of Mathematics, Kirtipur, TU
}

Article History: Received 14 May 2020; Reviewed 28 May 2020; Revised 30 June 2020; Accepted 10 July 2020

\begin{abstract}
This paper concerns our sustained efforts for introduction of M-fuzzy metric spaces and study their basic topological properties by introducing the Generalized metric space. As an application of this concept, we prove some Convergences, Cauchy and continuous properties related in M-fuzzy metric space and introduce some related examples in support of our results.
\end{abstract}

Keywords: D-metric space - continuous t-norm - convergence - M-Fuzzy metric space.

\section{INTRODUCTION}

A metric space is a non-empty set $\mathrm{X}$, associated with a function of two variables enabling us to measure the distance between points. In advanced mathematics, we need to find the distance not only between two numbers and vectors, but also between more complicated objects like sequences, sets and functions. In order to find an appropriate concept of a metric space, numerous approaches exist in this sphere. Thus, new notations of distance lead to new notations of convergence and continuity. A numbers of generalization of a metric space have been discussed by many eminent mathematicians.

The foundation of fuzzy mathematics is laid by L. A. Zadeh (1965) with the introduction of fuzzy sets. This foundation represents a vagueness in everyday life. Subsequently several authors have applied various form general topology of fuzzy sets and 
developed the concepts of fuzzy space. Kramosil and Michalek (1975) introduced the concept of fuzzy metric space. George et al. (1994) modified the notation of fuzzy metric space with the help of continuous t-norms. A number of continuous and reciprocal continuous theorems have been developed numbers by various authors in fuzzy metric space by using concepts of compatible map, implicit relation, weakly compatible map and $\mathrm{R}$ weakly compatible maps. As there is a generalization in generalized metric space or D-metric space initiated by Dhage (1992). He proved some results on a self-map satisfying the contraction for complete and bounded complete D-metric spaces. Rhoades (1996) generalized Dhage's contractive condition by increasing the number of factors and proved the existence of unique fixed point of a self-map in D-metric space.

Recently, motivated by the concept of compatibility for metric space, Singh and Sharma (2002) introduced the concept of D-compatibility of maps in D- metric space and proved some related theorems using a contractive condition. Similarly, Sedghi and Shobe (2006) introduced the concept of M-fuzzy metric space and continuity in M-fuzzy metric space, which is based on D-metric space. So far as our work is concerned, (X, D) will denote a $\mathrm{D}$-metric space, $\mathrm{N}$ the set of natural numbers and $\mathrm{R}+$ the set of all positive real. The present paper generalizes the results of Sedghi and Shobe and also many authors existing in the literature.

\section{PRELIMINARIES}

In this section, we define some important definitions and results which are useful in sequel.

Definition 1.1 (Dhang, 1992): [1] Let $X$ be a non-empty set. A generalized metric space (Dmetric) on $X$ is a function $D: X^{3} \rightarrow R^{+}$which satisfies the following conditions for each $x, z, y$, $z, a \in X$

(i) $D(x, y, z) \geq 0$,

(ii) $D(x, y, z)=0$ if and only $x=y=z$,

(iii) $D(x, y, z)=D(p\{x, y, z\})$, (symmetry) where $p$ is a permutation function, (i.e. $D(x, y$, $z)=D(y, z, x)=D(z, x, y))$.

(iv) $D(x, y, z) \leq D(x, y, a)+D(a, z, z)$.

The pair $(X, D)$ is called a generalized metric (or $D$-metric) space. 
Such functions are illustrated as

(a) $D(x, y, z)=\max \{d(x, y), d(y, z), d(z, x)\}$,

(b) $D(x, y, z)=d(x, y)+d(y, z)+d(z, x)$. where $D$ is the ordinary metric on $X$.

(c) If $X=R^{n}$, then we define as

$\|\cdot\|_{p}: R^{n} \rightarrow R$ by $\left\|\left(x_{1}, x_{2}, \ldots x_{n}\right)\right\|_{\mathrm{p}}=\left(\left|x_{1}\right|^{p}+\left|x_{2}\right|^{p}+\ldots\left|x_{n}\right|^{p}\right) \quad$ for all $\left(x_{1}, x_{2}, \ldots x_{n}\right) \in R^{n}$ and $x_{1}, x_{2}, \ldots x_{n} \in R$.

For $1 \leq p<\infty$, let us define $D(x, y, z)=\left(\|x-y\|^{p}+\left(\|y-z\|^{p}++\left(\|x-x\|^{p}\right) \frac{1}{p}\right.\right.$.

(d) if $X=R^{+}$then we define

$$
D(x, y, z)\left\{\begin{array}{l}
0 \text { if } x=y=z \\
\max \{x, y, z\} \text { otherwise, }
\end{array}\right.
$$

Remark (Dhang, 1992): In a $D$-metric space, we can prove that

$D(x, x, y)=D(x, y, y)$, as

(a) $D(x, x, y) \leq D(x, x, x)+D(x, y, y)=D(x, y, y)$ and similarly

(b) $D(y, y, x) \leq D(y, y, y)+D(y, x, x)=D(y, x, x)$

Hence from above $(a)$ and $(b)$ we have $D(x, x, y)=D(x, y, y)$.

Definition 1.2 (Dhang, 1992): Let $(X, D)$ be a $D$-metric space, then we define a ball $B_{D}(x, r)$ for $r>0$, with centre $c$ and radius $r$ as

$$
B_{D}(c, r)=\{x \in X: D(c, x, x)<r\} \quad \text { is called an open ball. }
$$

Example 1.3 (Dhang, 1992): Let $X=R$. and $D(x, y, z)=|x-y|+|y-z|+|z-x|$ for all $x, y, z \in R$. Then

$$
\begin{aligned}
& B_{D}(1,3)=\{y \in R: D(1, y, y, y)<3\}=\{y \in R:|y-1|+|y-1|+|y-1|<3\} \\
& =\{y \in R:|y-1|<1\}=(0,3) .
\end{aligned}
$$

Definition 1.4 (Sedghi \& Shobe, 2006): A sequence $\left\{x_{n}\right\}$ in $D$-metric space $(X, D)$ is said to converge to $x \in X$ if for each and $\in>0$ there exists $n_{0} \in N$ such that

$$
D\left(x, x, x_{n}\right)<\in \quad \text { for all } n \geq n_{0} .
$$

Similarly it is called Cauchy sequence if for each $\in>0$ and there exists $n_{0} \in N$ such that

$$
X\left(x_{n}, x_{n}, x_{m}\right)<\in \quad \text { for each } m, n \geq n_{0} .
$$

The $D$-metric space $(X, D)$ is said to be complete if for every Cauchy sequence is convergent. Theorem 1.5 (Sedghi \& Shobe, 2006): Let $(X, D)$ be a $D$-metric space. If sequence $\left\{x_{n}\right\}$ in $X$ converges to $\mathrm{x}$, then $\mathrm{x}$ is unique. 
Proof: Let $x_{n} \rightarrow y$ and $x \neq y$. Since $\left\{x_{n}\right\} x$ converges to $x$ and $y$, for each $\in>0$ there exists $n_{1} \in N$ such that for every $n \geq n_{1} \Rightarrow D\left(x, x, x_{n}\right)<\frac{\epsilon}{2}$ and $n_{2} \in N$ such that for every $n_{1} \geq n_{2}$ $\in N$ such that for every $n_{1} \geq n_{2} \Rightarrow D\left(y, y, x_{n}\right)<\frac{\epsilon}{2}$.

If we suppose $n_{0}=\max \left\{n_{1}, n_{2}\right\}$, then for every $n \geq n_{0}$, by triangle inequality we have $D(x, x$, $y) \leq D\left(x, x, x_{n}\right)+D\left(x_{n}, y, y\right)<\frac{\epsilon}{2}+\frac{\epsilon}{2}=\epsilon$.

Hence $D(x, x, y)=0$ which is a contradiction. So $x=y$.

Theorem 1.5 (Zadeh, 1965): Let $(X, D)$ be a $D$-metric space. If sequence $\left\{x_{n}\right\}$ in $X$ converges to $x$, then sequence $\left[x_{n}\right\}$ is a Cauchy sequence.

Proof: Since $x_{n} \rightarrow x$ for each $\in>0$ there exists $n_{1} \in N$ such for every $n \geq n_{1} \Rightarrow D\left(x_{n}, x_{n}, x\right)$

$<\frac{\epsilon}{2}$ and $n_{2} \in N$ such that for every $m \geq n_{2} \Rightarrow D\left(x, x_{m}, x_{m}\right)<\frac{\epsilon}{2}$

If we assume that $n_{0}=\max \left\{n_{1}, n_{2}\right\}$, then for every $n, m \geq n_{0}$ then by triangle inequity we have,

$$
D\left(x_{n}, x_{n}, x_{m}\right) \leq D\left(x_{n}, x_{n}, x_{n}\right)+D\left(x, x_{m}, x_{m}\right)<\frac{\epsilon}{2}+\frac{\epsilon}{2}=\epsilon \text {. }
$$

Hence $\left\{x_{n}\right\}$ is a Cauchy sequence.

Definition 1.6 (George \& Veeramani, 1994): A binary operation $*:[0,1] \times[0,1] \rightarrow[0,1]$ is continuous triangular norm (t-norm) if for all $a, b, c \in[0,1]$, the following conditions are satisfied.

(i) $a * 1=\mathrm{a}$

(ii) $a * b=b * a$

(iii) If $\mathrm{a} \leq \mathrm{c}$ and $b \leq d$ then $a * b \leq c * d$

(iv) $a *(b * c)=(a * b) * c$

(v) $*$ is continuous.

Here, $T_{p}(a, b)=a . b, T_{m}(a, b)=\min (a, b)$ and $T_{L}(a, b)=\max (a+b-1,0)$ are some examples of continuous t-norm.

Definition 1.7 (Sing \& Sharma, 2002): A 3-tuple $(X, M, *)$ is said to be a $M$-fuzzy metric space if $X$ is an arbitrary set, $*$ is a continuous t-norm and $M$ is a fuzzy set on $X^{3} \times(0, \infty)$ satisfying the following conditions for each $x, y, z, a \in X$, 
(i) $M(x, y, z, t)>0$,

(ii) $M(x, y, z, t)=1$ for all $\mathrm{t}>0$ if and only if $x=y=z$

(iii) $M(x, y, z, t)=M(p ;\{x, y, z\}, t)$ (symmetric) where $p$ is a permutation function

(iv) $\quad M(x, y, a, t) * M(a, z, z, s) \leq M(x, y, z, t+s)$ for all $t, s>0$

(v) $M(z, y, z,):.(0, \infty) \rightarrow[0,1]$ is continuous.

Remarks 1.8 (Sing \&Sharma, 2002): Let $(X, M, *)$ be a $M$-fuzzy metric space. We assert that for every $t>0 M(x, x, y, t)=\mathrm{M}(x, y, y, t)$.

For every $\in>0$ by triangle inequality we have

(i) $M(x, x, y, \in+t) \geq M(x, x, x, \in) * M(x, y, y, t)=M(x, y, y, t)$

(ii) $M(y, y, x, \in+t) \geq M(y, y, y, \in) * M(y, x, x, t)=M)(y, x, x, y, t)=M(x, y, y, t)$.

Now taking limit as $\in \rightarrow 0$ from (i) and (ii) we obtain $M(x, x, y, t)=M(x, y, y, t)$.

Open ball and convergence in M-fuzzy metric space (Sedghi \& Shobe, 2006):

Let $(X, M, *)$ be a M-fuzzy metric space. For $t>0$, we define an open ball

$B_{M}(x, r, t)$ with centre $x \in X$ and radius $0<r<1$ is defined

$$
B_{M}(x, r, t)=\{y \in X: M(x, y, y, t)>1-r\}
$$

As a consequent

A subset $A$ of $X$ is called open set if for each $x \in A$ there exists $t>0$ and $0<r<1$ such that $B_{M}(x, r, t) \subseteq A$.

A sequence $\left\{x_{n}\right\}$ in $X$ converges to $x$ if and only if $M\left(x, x, x_{n}, t\right) \rightarrow 0$ as $n \rightarrow \infty$, for each $t>$ 0 .

Similarly it is called a Cauchy sequence if for each $0<\in<1$ and $>0$, there exists $n_{0} \in N$ such that $M\left(x_{n}, x_{n}, x_{m}, t\right)>1-\in$ for each $m, n \geq n_{0}$.

The $M$-fuzzy metric space $(X, M, *)$ is said to be compete if every Cauchy sequence is convergent.

Example 1.9 (Sedghi \& Shobe, 2006): Let $X$ be an non empty set and $D$ is the $D$-metric space on $X$.

Let us denote $a * b=a . b$, for all $a, b \in[0,1]$. For each $t \in(0, \infty)$ and define

$$
M(x, y, z, t)=\frac{t}{t+D(x, y, z)}
$$


for all $x, y, z \in X$. Then it is easy to see that $(X, M, *)$ is a M-fuzzy metric space.

Theorem 1.10 (Sedghi \& Shobe, 2006): Let $(X, M, *)$ is a fuzzy metric space. If we define $M$ : $X^{3} \times(0, \infty) \rightarrow[0,1]$ by

$$
M(x, y, z, t)=M(x, y, t) * M(y, z, t) * M(z, x, t)
$$

for very $x, y, z \in X$, then $(X, M, *)$ is a $M$-fuzzy metric space.

Proof:

(1) It is easy to see that for every $x, y, z \in X, M(x, y, z, t)>0$ for all $t>0$

(2) $M(x, y, z, t)=1$, if and only if $M(x, y, t)=M(y, z, t)=M(z, x, t)=1$ if and only if $x=y=z$

(3) $M(x, y, z, t)=M(p\{x, y, z\}, t)$, where $p$ is a permutation function.

(4) $M(x, y, z, t+s)=M(x, y, t+s) * M(\mathrm{y}, z, t+s) * M(z, x, t+s)$

$\geq M(x, y, t) * \mathrm{M}(y, a, t) * M(a, z, s) * M(z, a, s) * M(a, x, t)$

$=M(x, y, a, t) * M(a, z, s) * M(z, a, s) * M(z, z, s)$

$=M(x, y, a, t) * M(a, z, z, s)$

for every s $>0$.

Lemma 1.11 (Sedghi \& Shobe, 2006): Let (X, $M, *)$ be a $M$-fuzzy metric space. Then $M(x, y$, $z, t)$ is non decreasing with respect to $t$, for all $x, y, z \in \mathrm{X}$

Proof: By definition of $M$-fuzzy metric space for each $x, y, z, a \in X$ and $t, s>0$ we have $M(x, y, a, t) * M(a, z, z, s) \leq M(x, y, z, t+s)$

Let us set $a=z$ we get $M(x, y, z, z, t) * M(z, z, z, s) \leq M(\mathrm{x}, \mathrm{y}, \mathrm{z}, \mathrm{t}+\mathrm{s})$, which gives $M(z, y$, $z, t+s) \geq M(x, y, z, t)$.

Definition 1.12 (Sedghi \& Shobe, 2006): Let $A$ and $B$ be two self mappings of a $M$-fuzzy metric space $(X, M, *)$ we say that $A$ and $B$ satisfy a property, if there exists a sequence $\left\{x_{n}\right\}$ such that

$\lim _{n \rightarrow \infty} M\left(A x_{n}, u, u, t\right)=\lim _{n \rightarrow \infty} M\left(B x_{n}, u, u, t\right)=1 \ldots(*)$

for some $u \in X$ and $r>0$. 
Example 1.13 (Sedghi \& Shobe, 2006): Let $X=R$ and $M(x, y, z, t)=$

$$
\frac{t}{t+|x-y|+|y-z|+|z-x|}
$$

for every $x, \mathrm{y}, z \in X$ and $t>0$

Let $A$ and $B$ be defined as $A x=2 \mathrm{x}+1, \mathrm{~B} x=x+2$.

Consider the sequence $x_{n}=\frac{1}{n}+1, n=1,2, \ldots$, then we have

$$
\lim _{n \rightarrow \infty} M\left(A x_{n}, 3,3, t\right)=\lim _{n \rightarrow \infty} M\left(B x_{n}, 3,3, t\right)=1
$$

for every $t>o$. Then $A$ and $B$ satisfying the property (*)

\section{MAIN RESULTS AND CONCLUSION}

Here by introducing the concept of continuous function on the domain $X^{2} \times(0, \infty)$ of $M$-fuzzy metric space, we establish the result of continuity of $M$ on the given domain by changing some parameters in the theorem of Sedghi [6]. Also using the definition of Mohd R., [2008], we show the relation between $M$-convergent and $M$-Cauchy sequence.

Definition 1.14 Let $(X, M, *)$ be a $M$-fuzzy metric space. Then $M$ is said to be continuous function on $X^{3} \times(0, \infty)$ if

$\lim _{n \rightarrow \infty} M\left(x_{n}, y_{n}, z_{n}, t_{n}\right)=M(x, y, z, t)$

Whenever a sequence $\left\{\left(x_{\mathrm{n}}, y_{\mathrm{n}}, z_{\mathrm{n}}, t_{n}\right)\right\}$ in $X^{3} \times(0, \infty)$ converges to a point $(x, y, z, \mathrm{t}) \in X^{3} \times(0$, $\infty)$ i.e.

$\lim _{n \rightarrow \infty} x_{n}=x, \lim _{n \rightarrow \infty} y_{n}=y, \lim _{n \rightarrow \infty} z_{n}=z$ and

$\lim _{n \rightarrow \infty} M\left(x, y, z, t_{n}\right)=M(x, y, z, t)$.

Now, we shall investigate the results that generalizes theorem 1.15 by S. Dedghi and N. Shobe (2006), by introducing some new parameters. 
Theorem 1.15: Let $(X, M, *)$ be a $M$-fuzzy metric space. Then $M$ is continuous function on $X^{3}$ $\times(0, \infty)$.

Proof: Let $x, y, z \in X$ and $t>0$, And let $\left(\mathrm{x}_{n}^{\prime}, y_{n}^{\prime}, z_{n}^{\prime}, t_{n}^{\prime}\right)$ is a sequence in [0,1], there is subsequence $\left(x_{n}, y_{n}, z_{n}, t_{n}\right)$ be sequence in $X^{3} \times(0, \infty)$ that $M$-converges to $(x, y, z, t)$.

Since $M\left(\mathrm{x}_{n}^{\prime}, y_{n}^{\prime}, z_{n}^{\prime}, t_{n}^{\prime}\right)$ is a sequence in $[0,1]$, there is subsequence $\left(x_{n}, y_{n}, z_{n}, t_{n}\right)$ of sequence $\left(M\left(\mathrm{x}_{n}^{\prime}, y_{n}^{\prime}, z_{n}^{\prime}, t_{n}^{\prime}\right)\right)$ such that sequence $\left(M\left(x_{n}, y_{n}, z_{n}, t_{n}\right)\right)$ converges to some point of $[0,1]$.

Fix $\delta>0$ such that $\delta=\frac{t}{2}$. Then, there is $n_{0} \in N$ such that $\left|t-t_{n}\right|<\delta$ for every $n \geq n_{0}$. Hence,

$\left(M\left(x_{n}, y_{n}, z_{n}, t_{n}\right)\right) \geq M\left(x_{n}, y_{n}, z_{n}, t-\delta\right)$

$\geq M\left(x_{n}, y_{n}, z, t-\frac{2 \delta}{3}\right) * M\left(z, z_{n}, z_{n}, \frac{-\delta}{3}\right)$

$\geq M\left(x_{n}, z, y, t-2 \delta\right) * M\left(y, y_{n}, y_{n}, \frac{\delta}{2}\right) * M\left(z, z_{n}, z_{n}, \frac{\delta}{2}\right)$

$\geq M\left(x, y, z, t-\frac{5 \delta}{2}\right) * M\left(x, x_{n}, x_{n}, \frac{\delta}{2}\right) * \mathrm{M}\left(y, y_{n} y_{n}, \frac{\delta}{2}\right) * M \quad\left(z, z_{n}, z_{n}, \frac{\delta}{2}\right)$

And

$\mathrm{M}(\mathrm{x}, \mathrm{y}, \mathrm{z}, \mathrm{t}+2 \delta) \geq \mathrm{M}\left(\mathrm{x}, \mathrm{y}, \mathrm{z}, \mathrm{t}_{\mathrm{n}}+\delta\right)$

$$
\begin{aligned}
& \geq M\left(x, y, z_{n}, t_{n}+\frac{\delta}{2}\right) * M\left(z_{n}, z, z, \frac{\delta}{2}\right) \\
& \geq M\left(x, z_{n}, y_{n}, t_{n}+\frac{3 \delta}{5}\right) * M\left(y_{n}, y, y, \frac{\delta}{5}\right) * M\left(z_{n}, z, z, \frac{\delta}{5}\right)
\end{aligned}
$$




$$
\begin{array}{cc}
\geq M\left(z_{n}, y_{n}, x_{n}, t_{n}+\frac{2 \delta}{5}\right) * & M\left(x_{n}, x, x, \frac{\delta}{5}\right) * M\left(y_{n}, y, y, \frac{\delta}{5}\right) * \\
M\left(z_{n}, z, z, \frac{\delta}{5}\right) & \text { for all } n \geq n_{0}
\end{array}
$$

By taking limit as $n \rightarrow \infty$, we have,

$\lim _{n \rightarrow \infty} M\left(x_{n}, y_{n}, z_{n}, t_{n}+\frac{2 \delta}{5}\right) \geq M(x, y, z, t-2 \delta) * 1 * 1 * 1=M(x, y, z, t-2 \delta)$

and $M(x, y, z, t+2 \delta) \geq M\left(x_{n}, y_{n}, z_{n}, t_{n}+\frac{2 \delta}{5}\right) 1 * 1 * 1=M\left(x_{n}, y_{n}, z_{n}, t_{n}+\frac{2 \delta}{5}\right)$

Now, taking as limit as $\delta \rightarrow 0$ we conclude that

$\lim$

$\lim _{n \rightarrow \infty} M\left(x_{n}, y_{n}, z_{n}, t_{n}\right)=M(x, y, z, t)$.

Therefore, $M$ is continuous on $X^{3} \times(0, \infty)$.

Definition 1.16 (Mohd, 2008): A sequence $\left\{x_{n}\right\}$ in $M$-fuzzy metric space $(X, M, *)$ is sai to be $M$-converge to a point $x_{0} \in X$ if

$\lim$

$\lim _{m, n \rightarrow \infty} M\left(x_{m}, x_{n}, t\right)=1$, for all $m, n>0, t>0$

Now, we show the equivalence of the definition of convergent and Cauchy sequence in $M$ fuzzy metric space given by Abdolrahman R., in 2005.

Theorem 1.17 Let $(X, M, *)$ be an $M$-fuzzy metric space and $\left\{\mathrm{x}_{\mathrm{n}}\right\}$ be a sequence in X. If $\left\{x_{n}\right\}$ is an $M$-convergent sequence, let us assume that it converges to $\mathrm{x}_{0}$ then for each $0<\epsilon<1$ and $t>0$, there exists $n_{0} \in N$ such that

$\lim _{n \rightarrow \infty} M\left(x_{n}, x_{0}, \frac{t}{2}\right)=\frac{1}{2}$, for each $t>0$

Proof: 
For all $m, n \geq n_{0}$, we have,

$$
\lim _{m, n \rightarrow \infty} M\left(x_{n}, x_{m}, t\right)=M\left(x_{n}, x_{0}, \frac{t}{2}\right) * M\left(x_{m}, x_{0}, \frac{t}{2}\right)=\frac{1}{2} * \frac{1}{2}=1
$$

Hence $\left\{x_{n}\right\}$ is an $M$-Cauchy sequence in fuzzy metric space $(X, M, *)$.

But the converse is not always true, which is shown by flowing example. Let $\mathrm{X}=(0,1]$. If we consider $M$ as a usual $M$-fuzzy metric space on $R$ and $a * b=a b$, for all $a, b \in[0,1]$, then $((0,1], M, *)$ is an $M$-fuzzy metric space.

If we consider $x_{n}=\frac{1}{n}, n=1,2,3, \ldots$ in $=(0,1]$, then

$$
\lim _{n \rightarrow \infty} M\left(x_{n}, x_{0}, \mathrm{t}\right)=\lim _{n \rightarrow \infty}\left(\frac{1}{n}, x_{0}, t\right) \neq 1 .
$$

Which shows that $\left\{x_{n}\right\}$ is not an $M$-convergent sequence, but it is an $M$-Cauchy sequence.

\section{REFERENCES}

Dhang, B. C. (1992). Generalized metric spaces and mappings with fixed point. Bull. Calcutta Math. Sos. 84(4), 329-336.

George, A., \& Veeramani, P. (1994). On some results on fuzzy metric space. Fuzzy Sets Syst, 64, 395-404.

Gregori, V., \& Sapena, A. (2002). On fixed point theorem in fuzzy metric spaces. Fuzzy Sets and Sys, 125, 245-252.

Kramosil, I., \& Michalek, J. (1975). Fuzzy metric and stoical metric spaces. Kybernetica, 11, 326334.

Rhoades, B. E. (1996). A fixed point theorem for generalized metric spaces. Int. J. Math. Sci. 19, 145-153.

Sedghi, S., \& Shobe, N. (2006). Fixed point theorem in M-fuzzy metric space with property (E). Advances in Fuzzy Mathematics, 1(1), 55-65.

Sing, B., \& Sharma, R. K. (2002). Common fifed points via compatible maps in D-metric spaces. Rad. Mat., 11, 145-153.

Zadeh, L. A. (1965). Fuzzy sets. Inform and Control, 8, 407-422. 